\title{
Enhanced Degradation in Polycrystalline Silicon Thin-Film Transistors Under Dynamic Hot-Carrier Stress
}

\author{
Kow Ming Chang, Yuan Hung Chung, Gin Ming Lin, Chi Gun Deng, and Jian Hong Lin
}

\begin{abstract}
We address the mechanisms responsible for the enhanced degradation in the polysilicon thin-film transistors under dynamic hot-carrier stress. Unlike the monotonic decrease of maximum transconductance $\left(G_{m \max }\right)$ in static stress, $G_{m \text { max }}$ in dynamic stress is initially increased due to the channel shortening effect by holes injected into the gate oxide near the drain region and then decreased due to tail states generation at the gate oxide/channel interface and grain boundaries. The threshold voltage variations are dominated by two degradation mechanisms: 1) breaking of weak bonds and 2) breaking of strong bonds to obey the power-time dependence law with a slope of 0.4 . The degradation of the sub-threshold slope is attributed to intra-grain bulk states generation.
\end{abstract}

Index Terms-Channel shortening effect, intra-grain bulk states, polysilicon thin-film transistors (poly-Si TFTs), tail states, transconductance $\left(G_{\operatorname{m} \max }\right)$.

\section{INTRODUCTION}

$\mathbf{P}$ OLYSILICON thin-film transistors (poly-Si TFTs) have received increasing attention for application in active matrix liquid crystal displays (AMLCDs) and static random-access memories (SRAMs). Reliability is one of the major concerns, especially when a device is operated under high drain and high gate voltages. Hot carrier stress under high drain and high gate voltages decreases the transconductance, turn-on current, sub-threshold slope and increases the threshold voltage [1], [2]. Although the study of device aging with stress time under static hot-carrier stress has been systematically reported [3], to our knowledge, a study of the degradation in poly-Si TFT performance under dynamic hot-carrier stress has not been reported. Moreover, dynamic stress is closer to real operational conditions than static stress [4]. In this letter, we study the degradation mechanism by analyzing the evolution of device parameters including transconductance, threshold voltage, and sub-threshold slope.

\section{EXPERIMENT}

First, an undoped 100-nm-thick amorphous Si film was deposited on 500-nm-thick thermal oxide by decomposition of

Manuscript received June 7, 2001; revised July 27, 2001. This work was supported by the National Science Council of Taiwan, R.O.C., under Contract NSC89-2215-E009-089. The review of this letter was arranged by Editor T.-J. King.

The authors are with the Department of Electronics Engineering and Institute of Electronics of National Chiao-Tung University, Hsinchu, Taiwan, R.O.C (e-mail: kmchang@cc.nctu.edu.tw).

Publisher Item Identifier S 0741-3106(01)08863-2.
$\mathrm{Si}_{2} \mathrm{H}_{6}$ at $460{ }^{\circ} \mathrm{C}$ in a low-pressure chemical vapor deposition system and then crystallized at $600{ }^{\circ} \mathrm{C}$ for $24 \mathrm{~h}$. The measured average grain size is $1.1 \mu \mathrm{m}$. After defining the active channel region, a 50-nm-thick plasma-enhanced chemical vapor deposition $\mathrm{SiO}_{2}$ film was deposited at $350{ }^{\circ} \mathrm{C}$ to serve as the gate dielectric. A 300-nm-thick amorphous Si gate layer was then deposited and patterned. Source and drain regions were formed by ion implantation of $\mathrm{P}^{+}\left(5 \times 10^{15} \mathrm{~cm}^{-2}, 50 \mathrm{KeV}\right)$ and then activated at $600{ }^{\circ} \mathrm{C}$ for $12 \mathrm{~h}$. Finally, passivation oxide and metal layers were deposited and patterned. Channel length and width are $10 \mu \mathrm{m}$ and $5 \mu \mathrm{m}$, respectively. No hydrogenation step was performed on the poly-Si TFTs.

For dynamic stress, the drain is biased at $V_{\mathrm{DS}}=22 \mathrm{~V}$ and the gate bias $V_{\mathrm{GS}}$ is square waveform switching from $0 \mathrm{~V}$ to $20 \mathrm{~V}$ with rise time $100 \mathrm{~ns}$, fall time $100 \mathrm{~ns}$ and duty cycle 50\%. For static stress, $V_{\mathrm{DS}}=22 \mathrm{~V}$ and $V_{\mathrm{GS}}=20 \mathrm{~V}$ were used. Threshold voltage is extracted by extrapolation of the $I_{\mathrm{DS}}-V_{\mathrm{GS}}$ curve from the point of $G_{\mathrm{m} \max }$ in the linear regime ( $\left.V_{\mathrm{DS}}=0.1 \mathrm{~V}\right)$.

\section{RESUlTS AND DisCUSSION}

Fig. 1(a) and (b) show the different degradation behaviors of drain current and transconductance under static and dynamic stress, respectively. For static stress, $G_{\mathrm{m} \max }$ decreases monotonically with the increase of the stress time at $V_{\mathrm{GS}}$ in the range of $0 \mathrm{~V} \sim 17 \mathrm{~V}$ and remains unchanged for $V_{\mathrm{GS}}>17 \mathrm{~V}$. For dynamic stress, the curve of the transconductance shows a parallel shift in the positive direction, while $G_{\operatorname{mmax}}$ is initially increased and then it starts to decrease after $1000 \mathrm{~s}$ stressing.

Fig. 2 shows the degradation of the $G_{\operatorname{mmax}}$ defined as $-\Delta G_{\mathrm{m} \max } / G_{\mathrm{m} \max , \mathrm{o}}$, where $\Delta G_{\operatorname{mmax}}=G_{\operatorname{mmax}, \mathrm{s}}-$ $G_{\operatorname{mmax}, \mathrm{o}}, G_{\mathrm{m} \max , \mathrm{o}}$ is the initial $G_{\mathrm{m} \max }$ and $G_{\mathrm{m} \max , \mathrm{s}}$ is the $G_{\mathrm{m} \max }$ for each stress time. For dynamic stress, $G_{\operatorname{mmax}}$ initially increases, then saturates and finally starts to decrease after the turnaround point (as indicated by the arrow in Fig. 2). $G_{\mathrm{m} \max }$ rising is attributed to channel shortening effect by holes injected into the gate oxide region near drain [5]. In the ON state, impact ionization will occur to generate hot electrons and hot holes near the drain. Once the device is switched from ON state to OFF state, the channel electrons will be repelled quickly into the drain side to gain additional energy and cause serious impact ionization near the drain. During the OFF state stress period, the electric field near the drain is very suitable $\left(V_{\mathrm{GS}}-V_{\mathrm{DS}}=-22 \mathrm{~V}\right)$ for holes injecting into the gate oxide. When the injected holes reach saturation at the turnaround point, tail states generation in the grain boundaries and gate 


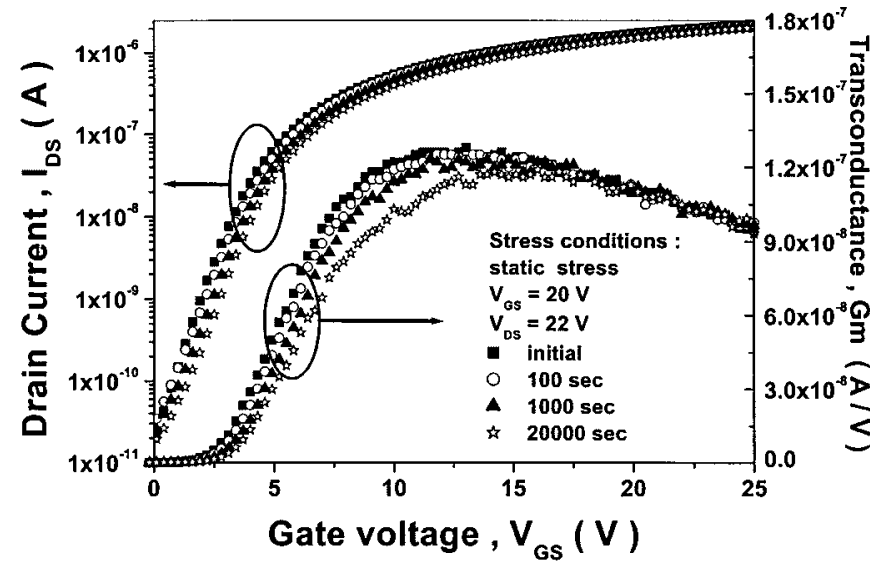

(a)

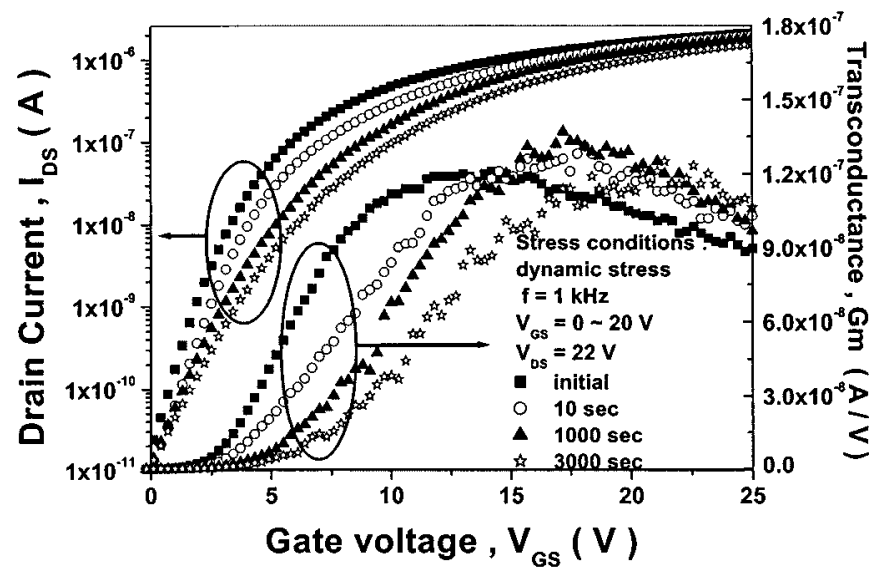

(b)

Fig. 1. Degradation of drain current and trans-conductance by (a) static stress and (b) dynamic stress.

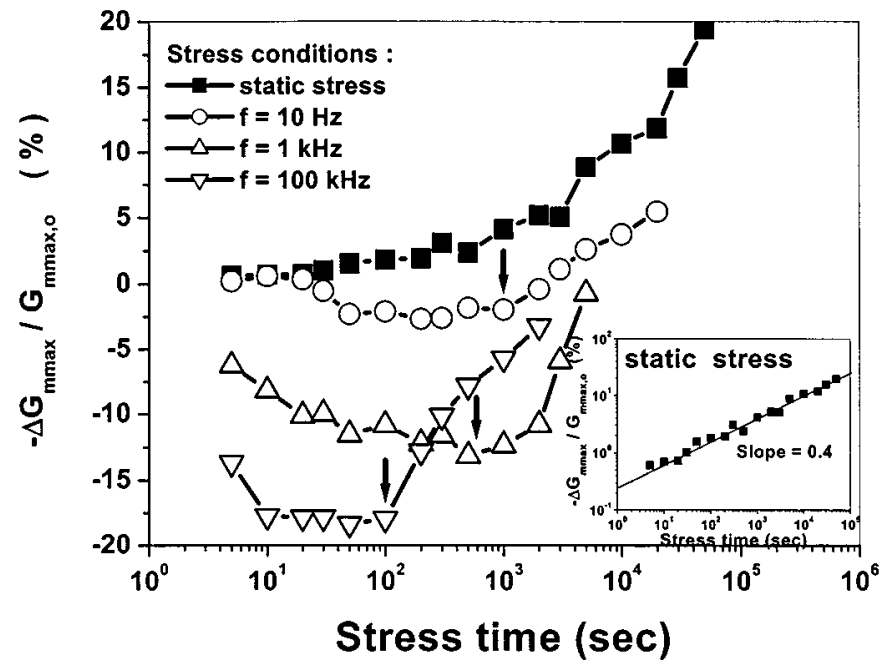

Fig. 2. Degradation of maximum trans-conductance as a function of the stress time under static and dynamic stress.

oxide/channel interface start to dominate $G_{\operatorname{mmax}}$ degradation [3]. As the stress frequency increases, hole concentration will increase [6] and cause more serious channel shortening effect. However, for static stress, $G_{\operatorname{mmax}}$ decreases monotonically and the insert plot of Fig. 2 shows that $G_{\operatorname{mmax}}$ degradation

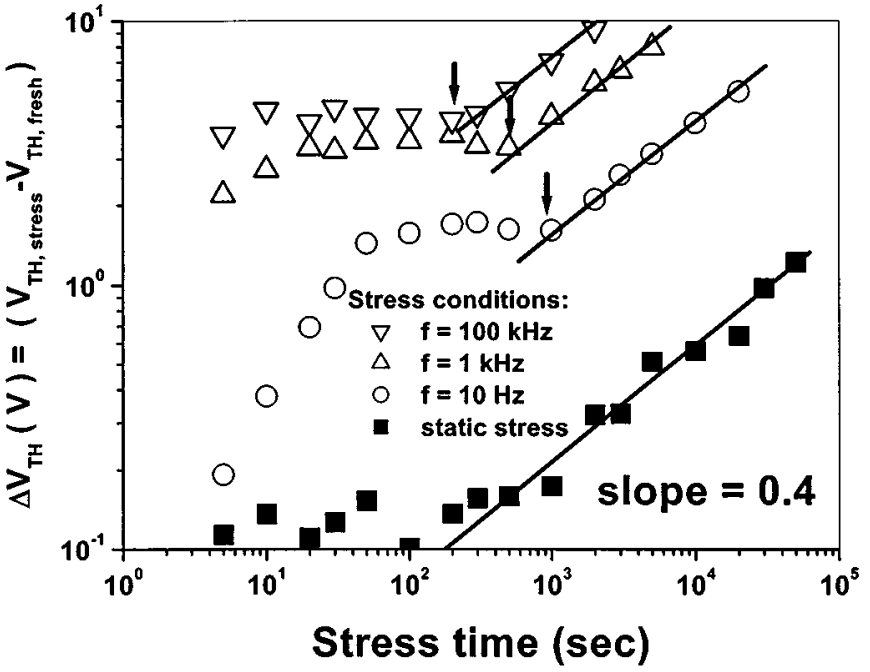

Fig. 3. Threshold voltage variations as a function of stress time under static and dynamic stress.



Fig. 4. Subthreshold slope variations as a function of stress time under static and dynamic stress.

exhibits a power-time dependence with a slope of 0.4 which is due to the tail states generation in the grain boundaries and at the gate oxide/channel interface [3], [7].

Fig. 3 shows the threshold voltage variations $\Delta V$ th after stressing. Clearly, $\Delta V$ th for static stress exhibits a power-time dependence with a slope of 0.4 and is much smaller than that of dynamic stress. For the dynamic stress, $\Delta V$ th first saturates (first stage) and then it shows a power-time dependent law with a slope of 0.4 (second stage). The degradation in the first stage is due to deep states generation in the grain boundaries and at the gate oxide/channel interface by breaking the weak $\mathrm{Si}-\mathrm{H}$ and $\mathrm{Si}-\mathrm{Si}$ bonds. Once all of the available weak bonds are broken, $\Delta V$ th saturates. The additional increase in $\Delta V$ th after the turnaround point indicated by the arrows in Fig. 3 is due to the strong $\mathrm{Si}-\mathrm{Si}$ bonds breaking [7], [8].

Fig. 4 shows the evolution of the sub-threshold slope variation $-\Delta S / S_{\mathrm{O}}$, where $\Delta S=S-S_{\mathrm{O}}, S_{\mathrm{O}}$ is the initial subthreshold slope and $S$ is the sub-threshold slope for each stress time. A power-time dependence with a slope of $0.18 \sim 0.25$ is 
observed for both static and dynamic stress. Unlike in laser-annealed poly-Si TFTs where the sub-threshold slope remains unchanged after stressing [3], in solid-phase crystallized poly-Si TFTs, the sub-threshold slope varies with stress time indicating that intra-grain bulk states are generated in the poly-Si film [9]. Because the poly-Si film was formed by solid-phase crystallization method in this letter, intra-grain bulk state density is much higher and $\mathrm{Si}-\mathrm{Si}$ bonds are weaker than those in laser-crystallized poly-Si film. Some more detailed study of the fall time and stress temperature effects on the device degradation is in progress.

\section{CONCLUSION}

Poly-Si TFT degradation mechanisms under dynamic hot-carrier stress were studied. $G_{\mathrm{m} \max }$ rising is due to channel shortening effect by hole injection into the gate oxide near the drain. As the stress frequency increases, the degradation becomes more serious. $\Delta V$ th is dominated by two degradation mechanisms: breaking of weak bonds initially and breaking of strong bonds to obey a power-time dependence law in the second stage. The degradation of the sub-threshold slope is attributed to intra-grain bulk states generation in the poly-Si film.

\section{REFERENCES}

[1] M. Hack, A. G. Lewis, and I. W. Wu, "Physical models for degradation effects in polysilicon thin-film transistors," IEEE Trans. Electron Devices, vol. 40, pp. 890-897, May 1993.

[2] F. V. Farmakis, C. A. Dimitriadis, J. Brini, G. Kamarinos, V. K. Gueorguiev, and Tz. E. Ivanov, "Interface state generation during electric stress in n-channel undoped hydrogenated polysilicon thin-film transistors," Electron Lett., vol. 34, no. 24, pp. 2356-2357, 1998.

[3] F. V. Farmakis, J. Brini, G. Kamarinos, and C. A. Dimitriadis, "Anomalous turn-on voltage degradation during hot-carrier stress in polycrystalline silicon thin-film transistors," IEEE Electron Device Lett., vol. 22 , pp. 74-76, Feb. 2001.

[4] Y. Uraoka, T. Hatayama, and T. Fuyuki, "Reliability evaluation method of low temperature poly-silicon TFT's using dynamic stress," in Proc. of the 2000 Int. Conf. on Microelectronic Test Structures, 2000, pp. $158-162$.

[5] F. V. Farmakis, J. Brini, G. Kamarinos, C. A. Dimitriadis, V. K. Gueorguiev, and Tz. E. Ivanov, "Electrical stress in N- and P- channel undoped-hydrogenated plolysilicon thin film transistors (TFT's)," in Int. Semiconductor Conf. CAS '99 Proc., vol. 1, 1999, pp. 157-160.

[6] A. G. Lewis, I. W. Wu, M. Hack, A. Chiang, and R. H. Bruce, "Degradation of polysilicon TFT's during dynamic stress," in in IEDM Tech Dig., 1991, pp. 575-578.

[7] G. A. Armstrong, S. Uppal, S. D. Brotherton, and J. R. Ayres, "Differentiation of effects due to grain boundary traps in laser annealed poly-S thin film transistors," Jpn. J. Appl. Phys., vol. 37, pp. 1271-1276, 1998.

[8] F. V. Farmakis, J. Brini, G. Kamarinos, C. T. Angelis, C. A. Dimitriadis, M. Miyasaka, and T. Ouisse, "Grain and grain-boundary control of the transfer characteristics of large-grain polycrystalline silicon thin-film transistors," Solid-State Electron, vol. 44, pp. 913-916, 2000.

[9] G. A. Armstrong, S. Uppal, S. D. Brotherton, and J. R. Ayres, "Modeling of laser-annealed polysilicon TFT characteristics," IEEE Electron Device Lett., vol. 18, pp. 315-318, July 1997. 